\title{
Stimulated Raman Spectra of Jet-Cooled Ethylene
}

\author{
L. Abad, D. Bermejo, R. Escribano, V.J. Herrero, J. Santos, I. Tanarro \\ Instituto de Estructura de la Materia, C.S.I.C., \\ Serrano 123, 28006 Madrid, Spain \\ G. D. Nivellini \\ Dipartimento di Chimica Fisica e Inorganica, \\ Viale Risorgimento 4, 40136 Bologna, Italy \\ L. Ramonat \\ Max Planck Institut für Strömungsforschung, \\ Bunsenstrasse 10, 37073 Göttingen, Germany
}

April 27, 1994

\begin{abstract}
We have recorded stimulated Raman spectra of the central region of the $\nu_{1}$ band of ethylene, both in a jet at temperatures down to $\sim 25 \mathrm{~K}$, and in a static cell at room temperature and at $\sim 173 \mathrm{~K}$. The rotational temperatures of the jet are estimated by computer simulation of the observed spectra. Only $\mathbf{Q}$ branches are recorded in this experiment. A vib-rotational analysis is performed and improved values are obtained for the band origin and the rotational constants of the $v_{1}=1$ state of this molecule. Some perturbations are found to affect the rotational levels of this state.
\end{abstract}




\section{INTRODUCTION}

The Raman spectrum of ethylene was studied some years ago by Foster, Hills and Jones [1] as a part of a thorough study [2-5] which set forth the general theory and treatment of the Raman spectra of asymmetric top molecules. Infrared studies of ethylene and of its deuterium and ${ }^{13} \mathrm{C}$ derivatives are frequent in the literature, but, as far as we know, only one further work has been published on the Raman spectra of $\mathrm{C}_{2} \mathrm{H}_{4}$, in spite of the fact that six of its fundamental vibrations are Raman active only, and information on the corresponding excited vibrational states can be obtained from infrared experiments only through the analysis of combination bands. The development of high resolution stimulated Raman techniques has enabled the recording of spectra of polarized Raman scattering of vib-rotational bands with near-Doppler resolution. These techniques were first applied to the very intense breathing vibration of spherical top molecules $[6-9]$, but more sophisticated experimental arrangements have allowed the extension of these methods to the recording of spectra of other molecules $[10-13]$. The only further publication on $\mathrm{C}_{2} \mathrm{H}_{4}$ mentioned above is that of Ref. [14], which contains, among several applications, a brief description of the CARS (Coherent Anti-Stokes Raman Scattering) spectrum of the $\nu_{2}$ fundamental band, recorded in a static cell and in a jet.

As is well known, polarized trace scattering corresponds to $\mathrm{Q}$ branches $(\Delta J=$ $0, \Delta K=0)$, giving rise to dense spectra, where most of the lines are blended. Besides, anisotropy scattering following $\Delta J= \pm 1, \pm 2$ selection rules, is usually at least one order of magnitude weaker than trace scattering, and the corresponding $\mathrm{O}, \mathrm{P}, \mathrm{R}$ and $\mathrm{S}$ transitions, which could be used to make up combination differences, are very often beyond the detection capabilities of the technique. Thus, the assignment of these spectra can be a difficult task. However, jet expansions of the molecular gas produce an outstanding cooling of the sample, which in turn remarkably simplifies the spectra as a consequence of the redistribution of the population within a few rotational levels. For a rotational temperature of $\sim 20 \mathrm{~K}$, the spectrum of a molecule like ethylene consists of about a dozen transitions. The assignment is almost straightforward in these cases, and this also facilitates the assignment of the rest of the spectrum.

We present in this paper a study of the symmetric $\mathrm{CH}_{2}$ stretching vibration of ethylene $\mathrm{C}_{2} \mathrm{H}_{4}$. We have recorded stimulated Raman spectra of this band at a range of temperatures: at room temperature and at $173 \mathrm{~K}$ in a static cell, and at different cold 
temperatures, reaching $\sim 25 \mathrm{~K}$, in a free jet expansion. These spectra are fully characterized. An analysis of the vib-rotational lines measured in the spectra is also presented. Similar to what is normally found in the analysis of infrared bands in this region, complications are present in the spectra, in the form of vib-rotational interactions with other close-lying vibrational states.

\section{EXPERIMENTAL}

The stimulated Raman loss (SRL) spectra of ethylene have been recorded in the region between 3017 and $3022 \mathrm{~cm}^{-1}$. The experimental arrangement for the SRL spectroscopic measurements is described in detail in Refs. [15-17], and only a brief description will be given here. A scheme of the setup is displayed in Fig. 1. The probe beam was provided by an $\mathrm{Ar}^{+}$laser (Spectra Physics 165) operating single mode at $488 \mathrm{~nm}$. The laser was frequency stabilized and locked to a hyperfine transition of ${ }^{130} \mathrm{Te}_{2}$ in a polarization spectroscopy configuration. The output signal had a bandwidth of less than $1 \mathrm{MHz}$ and a power of about $400 \mathrm{~mW}$. For the pump beam, the emission of a ring dye laser (Spectra Physics 380) operated with Rhodamine 110 was pulse amplified in a three stage amplifier, pumped by the second harmonic of a seeded Nd:YAG laser (Quanta Ray GCR-2) with an extended cavity. In the first two amplifying stages Rhodamine 6G was used, while the last one was operated with Rhodamine B. The output of the pump laser consisted in temporally and spatially Gaussian pulses of 12 ns with a bandwidth of about $2.5 \times 10^{-3} \mathrm{~cm}^{-1}$, limited by the Fourier transform of the temporal width of the pulses. The energy per pulse was about $3 \mathrm{~mJ}$ at a repetition rate of $14 \mathrm{~Hz}$. Pump and probe beams with parallel polarization were collinearly mixed by means of a dichroic mirror, and focused into the Raman cell or into the expansion chamber using a relatively large focal length lens, $f=500 \mathrm{~mm}$, in order to avoid significant broadening or shifting due to the ac Stark effect.

The room temperature spectra were recorded at a pressure of $12 \mathrm{mbar}$. Unblended lines showed a linewidth of $0.009 \mathrm{~cm}^{-1}$, close to the Doppler limit (about $0.007 \mathrm{~cm}^{-1}$ ). Some spectra were also recorded at $173 \mathrm{~K}$ by placing the cell into a melting iso-propanol bath.

The jets were formed either by expanding either pure ethylene or mixtures with He or Ar with which a stronger cooling was attained. The gases, at 5-7 bar pressures, were expanded through a solenoid actuated pulsed valve (General Valve 9-347-900) into a vacuum chamber. The circular exit of the valve, with a nominal diameter of $0.05 \mathrm{~cm}$, 
was extended into a piece of a copper tube, flattened at the end to form a slit, $0.012 \mathrm{~cm}$ wide and $0.85 \mathrm{~cm}$ long, to match the Rayleigh range of the focused beams, estimated to be $0.6 \mathrm{~cm}$. Gas pulses of about $500 \mu$ s duration were selected. The actual time profile of these pulses was monitored with a fast ionization gauge. Using the signal from this gauge the gas pulses were synchronized with the laser pulses. The background pressure in the chamber was kept in the $10^{-3}$ mbar range by means of a $1000 \mathrm{ls}^{-1}$ diffusion pump.

The use of a slit in the nozzle exit allowed a better interaction of the collinear pump and probe lasers with the sample, resulting in an increase of the measured signal. In addition, the experimental resolution of this arrangement, in which the expansion has parallel streamlines, is increased with respect to that achieved for a jet produced by a circular nozzle. In this last case, molecules in streamlines diverging from the axis of the expansion have relatively large velocity components in the direction of propagation of the lasers, which gives rise to a Doppler broadening of the lines. For our coldest planar jets, linewidths of about $0.005 \mathrm{~cm}^{-1}$ have been obtained.

\section{DESCRIPTION OF THE SPECTRA AND ANALYSIS}

The cell spectra are congested in the head of the band, in the region of the lower $K_{a}$ values, but show clear $J$ progressions for higher $K_{a}$ values. Fig. 2 displays the spectrum recorded in the cell at room temperature in the region between 3017 and $3022 \mathrm{~cm}^{-1}$. Some $J$ values corresponding to $K_{a}=6$ to 10 are indicated in the figure. The cooling attained in the supersonic expansions led to a simplification of the region corresponding to the lower $K_{a}$, which has been of great help for the assignments. Two of the jet spectra are shown in Fig. 3, together with computer simulations, which have been used to estimate the rotational temperature of the expansion. The jets formed in expansions of pure ethylene reached a cooling temperature of $\sim 40 \mathrm{~K}$, whereas free jets of mixtures of $16 \%$ ethylene in He cooled down to $\sim 25 \mathrm{~K}$. The distribution of rotational states appears to be approximately Boltzmann in all cases and the accuracy of the estimated temperatures is $\sim 5 \mathrm{~K}$. An evaluation of the translational temperature can also be performed from the Doppler width of the spectrum, estimated by deconvoluting the observed lineshape functions with the instrumental function. Temperatures calculated in this way agree approximately with the rotational temperatures estimated by the computer simulation scheme. 
The rotational assignment of the spectra was carried out from the more characteristic features found in each region. Thus, in the spectrum of the colder expansion in Fig. 3, we first recognized the $2_{02}$ and $4_{04}$ lines, because of their higher statistical weight (nuclear spin statistical weights for ethylene are: 7 for $K_{a}, K_{c}$ both even, 3 in any other case). The $K_{a}=2$ series was also identified, but lines of the $K_{a}=1$ series, which are split because of asymmetry, were harder to assign, since different choices are possible for the individual components. The spectrum of the $40 \mathrm{~K}$ expansion shown in Fig. 3 permitted us to extend the assignment to the first $J$ lines of the $K_{a}=3$ and 4 series. On the other hand, the spectrum at $173 \mathrm{~K}$ afforded the assignment of the $K_{a}=6,7$ and 8 , whereas the $K_{a} \geq 9$ series are best identified in the room temperature spectrum. The $K_{a}=5$ series was more difficult to assign, since the first and more intense lines of this set appear in one of the most congested zones in the higher temperature spectra, and the population of the corresponding levels is too small for being observed in the jet spectra. However, this series was finally assigned as the analysis of the rest of the data was proceeding and predictions from refined parameters were available.

We have carried out a vib-rotational analysis of the assigned transitions in order to refine the parameters of the $v_{1}=1$ state. The analysis was done in the $\mathrm{I}^{R}$ representation, using Watson's [18] Hamiltonian and the A-reduction. Accurate values of the rotational parameters of the ground state of this molecule are known from the ground state combination difference analysis of Cauuet et al [19], performed over 4300 differences arising from their infrared analysis of the $\nu_{7}$ band.

From the early stages of the analysis we found an anomalous behavior of the data: predictions to higher $K_{a}$ series performed with the parameters refined from low $K_{a}$ transitions, showed an increasing observed-calculated negative deviation. Attempts to include together all available data in a least squares fit lead to an unrealistic estimation of the centrifugal distortion constants. The only way to proceed in the fitting process was to use a damping scheme which scaled down the corrections to the centrifugal distortion constants before adding them to their values of the previous iteration cycle. We also had to liberate some of the cubic and one of the octic distortion constants in order to reproduce the observed data. Besides, transitions with $K_{a} \geq 9$ had to be left out of the fit, because of an apparent incompatibility with the rest of the data. The result of this fit is summarized in Table I.

The reason for the observed problems must be the presence of a set of levels close above those of $v_{1}=1$, belonging to a different vibrational state, and having the appropriate symmetry to interact with the former. Duncan and Hamilton [20] have obtained an improved harmonic force field for this molecule, from which they evaluated the Coriolis 
coupling constants among the fundamental vibrations. The most obvious state close to $v_{1}=1$ that can interact with it is the $v_{5}=1$ (of $B_{1 g}$ symmetry), situated around $50 \mathrm{~cm}^{-1}$ above. A possible Coriolis interaction between states of $A_{g}$ and $B_{1 g}$ symmetry, occurs for levels with $\Delta K_{a}= \pm 1$. Using the vib-rotational parameters of Foster et al [1], we have calculated the energy levels of the $v_{1}=1$ and $v_{5}=1$ states, and we have found that the energy gap between $K_{a}$ levels of $v_{1}=1$ and $K_{a}-1$ levels of $v_{5}=1$, which starts as negative, becomes smaller in absolute value with increasing $K_{a}$, and would be reversed in sign at $K_{a} \sim 9$. This is illustrated in Figure 4. Although the Coriolis parameter for this perturbation is small $\left(\zeta_{1,5}^{c}=0.05\right)$, a rough calculation of its effects gives a shift on the $K_{a}=9$ levels of $v_{1}=1$ of $\sim+0.04 \mathrm{~cm}^{-1}$, whereas the observed-calculated prediction with the parameters of Table I is $+0.07 \mathrm{~cm}^{-1}$. Thus, although this $\nu_{1} / \nu_{5}$ resonance could explain a good part of the observed effects, other perturbations must be present. Some candidates for such perturbations are the $v_{2}=v_{3}=1$ state $\left(A_{g}\right)$, observed at 2960 $\mathrm{cm}^{-1}[1]$, which can give a Fermi resonance with $v_{1}=1$; the $v_{6}=1, v_{8}=2$ state $\left(B_{1 g}\right)$, predicted at $3106 \mathrm{~cm}^{-1}$, which could also give a $c$-type Coriolis perturbation similar to that with $v_{5}=1$; and, more interestingly, the $v_{4}=v_{6}=v_{10}=1$ state $\left(B_{2 g}\right)$, predicted at $3075 \mathrm{~cm}^{-1}$, only $45 \mathrm{~cm}^{-1}$ above $\nu_{1}$. Interaction between $A_{g}$ and $B_{2 g}$ states would correspond to an $a$-type Coriolis resonance, giving large $K_{a}$-dependent matrix elements for the perturbation [20]. The complete study of all these perturbations would require a more extensive spectrum, including data on the $O, P, R$ and $S$ branches, not accessible in the present investigation.

Since the perturbations still remaining within the data set have not been explicitly taken into account, the standard deviation of the fit, which is about one third of the resolution of the observed spectrum, can be considered satisfactory. These perturbations also cause the sextic and octic distortion parameters of Table I to take unrealistic values, and they can only be taken as fitting parameters. The only point of reference for the band origin and the rotational constants of the $v_{1}=1$ state is the paper by Foster et al [1]. Although the uncertainties of the parameters are much smaller in the present work, owing to the higher resolution of the spectrum and the consequent higher precision of the data, it is interesting to compare the results of both investigations. Since the ground state constants used in each case are not the same, we have to compare the differences $\Delta=$ (ground state $-v_{1}$ state). This comparison is provided in the last two columns of Table I. The differences are matched well within the estimated uncertainties of the parameters for the $B$ and $C$ constant, but not for $A$ (discrepancy about 5 times larger than the quoted error for one standard deviation). This discrepancy may arise from the different value of the centrifugal distortion constants used in each case, or it may be a consequence of 
the intrinsic incompatibility of the data, due to the perturbation with $\nu_{5}$, and of the fact that different data sets have been used in Foster's analysis and the present one. Finally, it is also interesting to note from Table I the accurate value of the origin of the band obtained in this work, which was more difficult to achieve in Ref. [1], as Foster et al could not resolve the crowded ${ }^{Q} Q$ structure in the central region of the spectrum.

\section{Acknowledgment}

This work has been carried out with finantial support from the Spanish DGICYT, Projects PB91-0128 and PB90-0123. International collaboration was supported by a Cooperation Program between CSIC of Spain and CNR of Italy, and by the Integrated Action HA93-088 between the DGICYT (Spain) and the BMFT (Germany).

\section{References}

[1] R.B. Foster, G.W. Hills and W.J. Jones, Mol. Phys. 33, 1589 (1977).

[2] G.W. Hills, R.B. Foster and W.J. Jones, Mol. Phys. 33, 1571 (1977).

[3] G.W. Hills and W.J. Jones, Trans. Faraday II, 71, 812 (1975).

[4] G.W. Hills and W.J. Jones, Trans. Faraday II, 71, 826 (1975).

[5] G.W. Hills and W.J. Jones, Trans. Faraday II, 71, 835 (1975).

[6] A. Owyoung, C.W. Patterson and R.S. McDowell, Chem. Phys. Lett. 59, 156 (1978)..

[7] R.S. McDowell, C.W. Patterson and A. Owyoung, J. Chem. Phys. 72, 1071 (1980).

[8] P. Esherick, A. Owyoung and C.W. Patterson, J. Mol. Spectrosc. 86, 250 (1981).

[9] B. Lavorel, R. Saint-Loup, G. Pierre and H. Berger, J. Physique Lett. 45, L-295 (1984).

[10] P. Esherick, A. Owyoung and J. Pliva, J. Chem. Phys. 83, 3311 (1985).

[11] H. Frunder, R. Angstl, D. Illig, H.W. Schrötter and L. Lechuga-Fossat, Can. J. Physics 63, 1189 (1985).

[12] L.A. Rahn and D.A. Greenhalgh, J. Mol. Spectrosc. 119, 11 (1986). 
[13] R. Escribano, J.L. Domenech, P. Cancio, J. Ortigoso and D. Bermejo, J. Chem. Phys., in press.

[14] R.L. Byer, M. Duncan, E. Gustafson, P. Oesterlin, F. Konig, in Laser Spectroscopy V, Springer Ser. Opt. Sci., A.R.W. McKellar, T. Oka and B.P. Stoichoff, eds., pp. 233-241, Springer-Verlag, Berlin, 1981.

[15] Bermejo, J. Santos, P. Cancio, J.L. Domenech, C. Domingo, J.M. Orza, J. Ortigoso and R. Escribano, J. Raman Spectrosc. 21, 197 (1990).

[16] D. Bermejo, J. Santos, P. Cancio, J.M. Fernandez and S. Montero, J. Chem. Phys. 97. 7055 (1992).

[17] J. Santos, P. Cancio, J.L. Domenech, J. Rodriguez and D. Bermejo, Laser Chem. 12. 53 (1992).

[18] J.K.G. Watson, in "Vibrational Spectra and Structure" (J.R. Durig, Ed.), Vol. 6, Elsevier, 1977.

[19] I. Cauuet, J. Walrand, G. Blanquet, A. Valentin, L. Henry, Ch. Lambeau, M. de Vleeschouwer and A. Fayt, J. Mol. Spectrosc. 139, 191-214 (1990).

[20] J.L. Duncan and E. Hamilton, J. Mol. Struct. 76, 65-80 (1981).

[21] L. Nemes, J. Mol. Spectrosc. 72, 102-123 (1978). 
Table I. Vib-rotational parameters ${ }^{a}$ of the $v_{1}=1$ state of ${ }^{12} \mathrm{C}_{2} \mathrm{H}_{4}$ in the $A$-reduction.

\begin{tabular}{llll}
\hline & & $\Delta^{b}$ & $\Delta$ (Ref. 1) \\
\hline A & $4.80511(11)$ & 0.05950 & $0.0493(16)$ \\
$\mathrm{B}$ & $0.996429(23)$ & 0.00463 & $0.001(4)$ \\
$\mathrm{C}$ & $0.824911(18)$ & 0.00313 & $0.002(5)$ \\
$\Delta_{J} \times 10^{6}$ & $1.399(45)$ & & \\
$\Delta_{J K} \times 10^{5}$ & $0.849(99)$ & & \\
$\Delta_{K} \times 10^{4}$ & $0.792(34)$ & & \\
$\delta_{J} \times 10^{6}$ & $0.278(28)$ & & \\
$\delta_{K} \times 10^{4}$ & $0.100(25)$ & & \\
$\Phi_{J} \times 10^{12}$ & -1.668 & & \\
$\Phi_{J K} \times 10^{6}$ & $-0.1009(25)$ & & \\
$\Phi_{K J} \times 10^{6}$ & $-0.758(17)$ & & \\
$\Phi_{K} \times 10^{6}$ & $-1.048(35)$ & & \\
$\mathrm{L}_{J K K} \times 10^{9}$ & $1.402(38)$ & & \\
$\nu_{0}$ & $3021.85539(78)$ & & \\
$r m s$ & 0.0030 & & \\
$\mathrm{~N}$. of data & 230. & & \\
\end{tabular}

${ }^{a}$ All parameters in $\mathrm{cm}^{-1}$. Uncertainties quoted in units of the least significant digit. Parameters without uncertainty are fixed to their ground state value.

${ }^{b}$ Values of $\Delta$ indicate parameter differences as (ground state $-v_{1}=1$ state). 


\section{Captions to figures}

Figure 1. A scheme of the experimental setup used to obtain the Stimulated Raman Spectra. The abbreviations used have the following meaning: PID, non-inverting Proportional amplifier, Integrator, Differentiator; HVA, High Voltage Amplifier; OSC, oscilloscope; AOM, Acousto-Optic Modulator; SHG, Second Harmonic Generator; FPI, Fabry-Perot Interferometer; An., analyzer; Pol., polarizer; SP, Spatial Filter.

Figure 2. Stimulated Raman spectrum of ethylene at room temperature in the region of the band origin. Raman intensities are shown in arbitray units, although the scale is the same for all three regions displayed. Assignments are indicated for some of the more obvious transitions with $K_{a} \geq 6$.

Figure 3. Spectra taken in jet expansions. Left: ethylene diluted to $16 \%$ in He, laser focused at $4 \mathrm{~mm}$ from the jet exit. Right: pure ethylene expansion, $6 \mathrm{~mm}$ distance from the exit. In either case, the upper traces are simulated spectra where the more important assignments are shown. Simulations have been used to estimate the rotational temperature of the expansion, with an estimated accuracy of $5 \mathrm{~K}$.

Figure 4. Rotational energy levels of the $v_{1}=1$ (left) and $v_{5}=1$ (right) vibrational states of ethylene calculated using the parameters of Foster et al [1]. Levels are plotted for $J=15$ as an example. Level separation is $\sim 28 \mathrm{~cm}^{-1}$ for $K_{a}=5$ and $\sim-2 \mathrm{~cm}^{-1}$ for $K_{a}=9$ of $v_{1}=1$. 


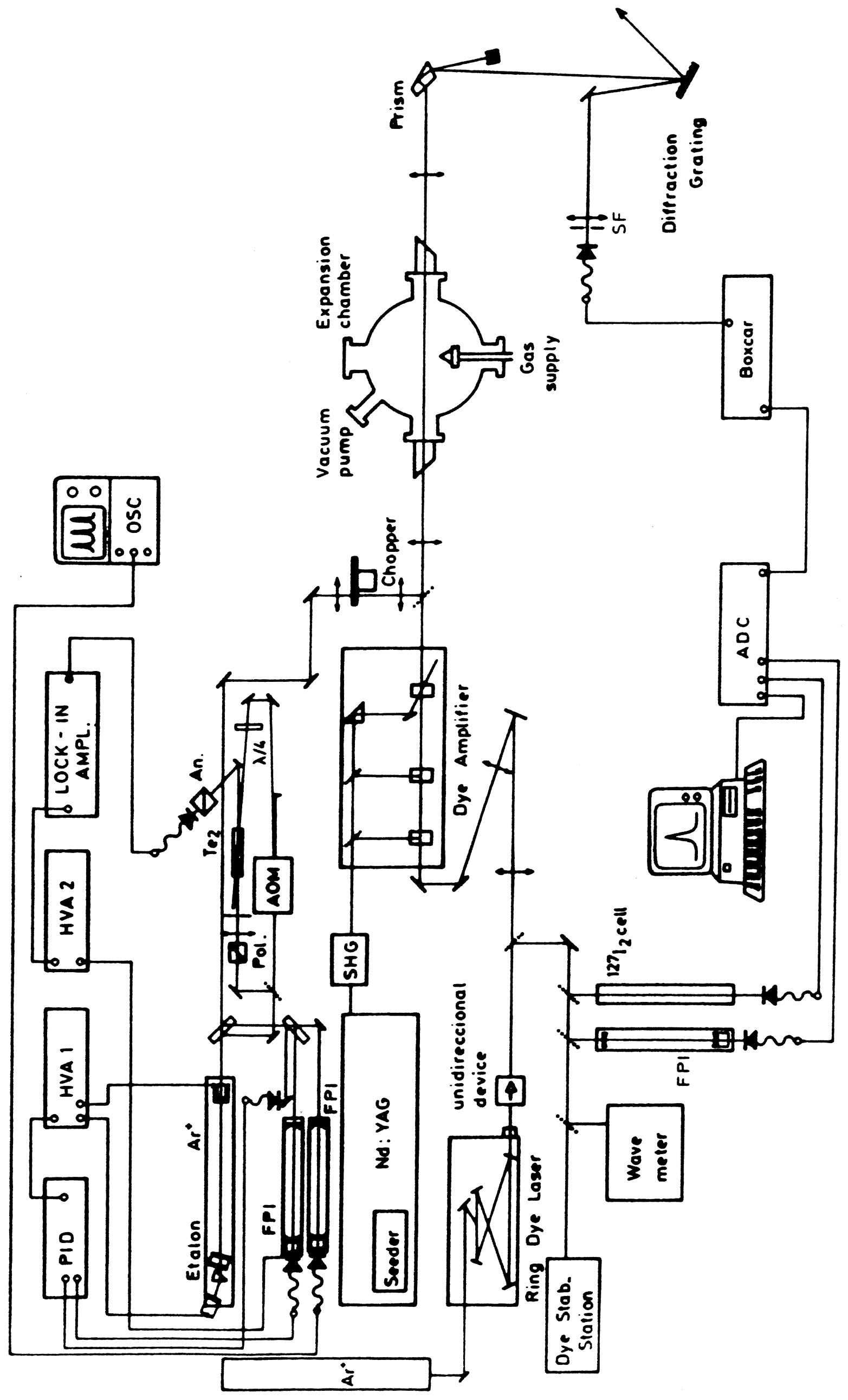



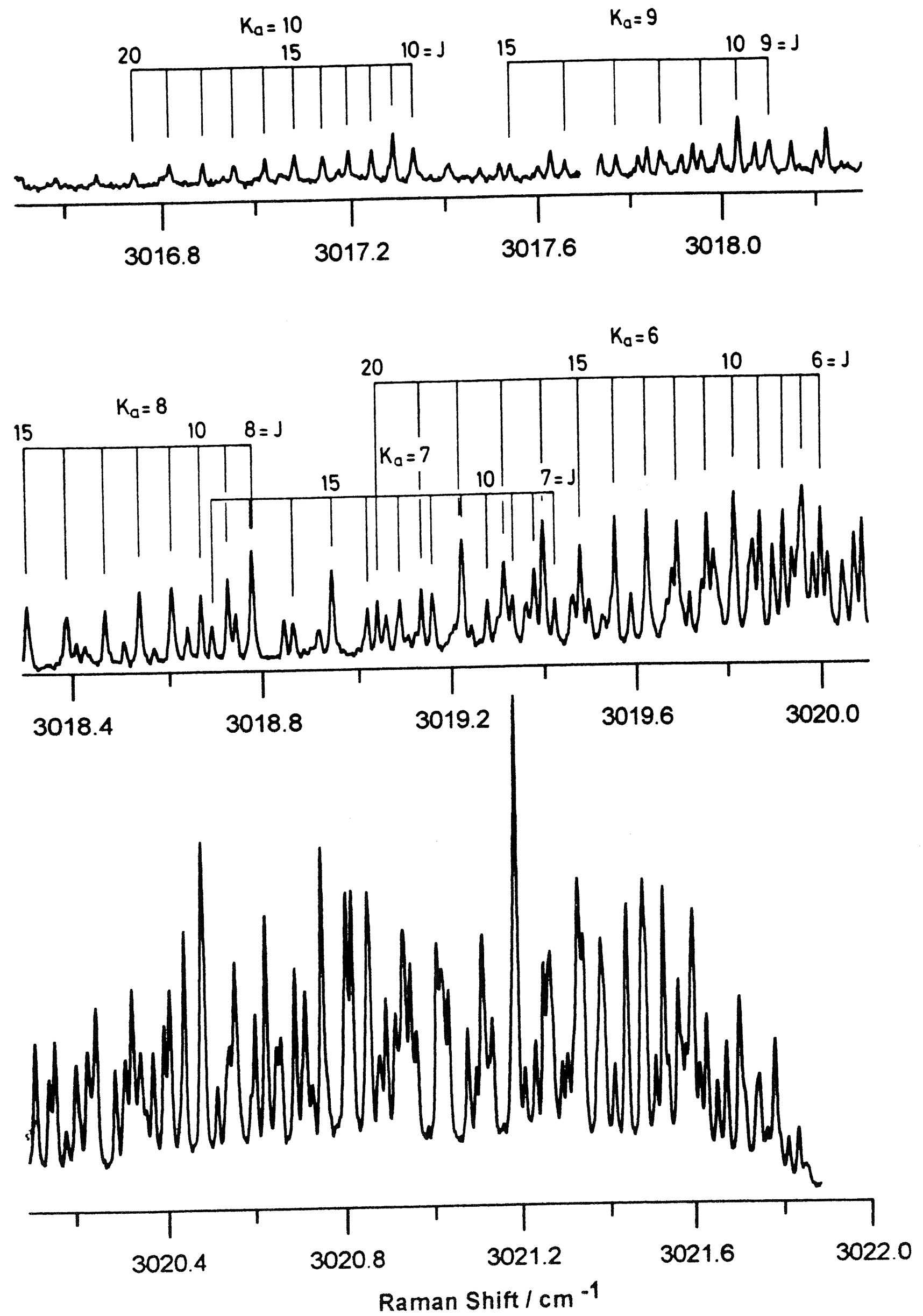
Calc.

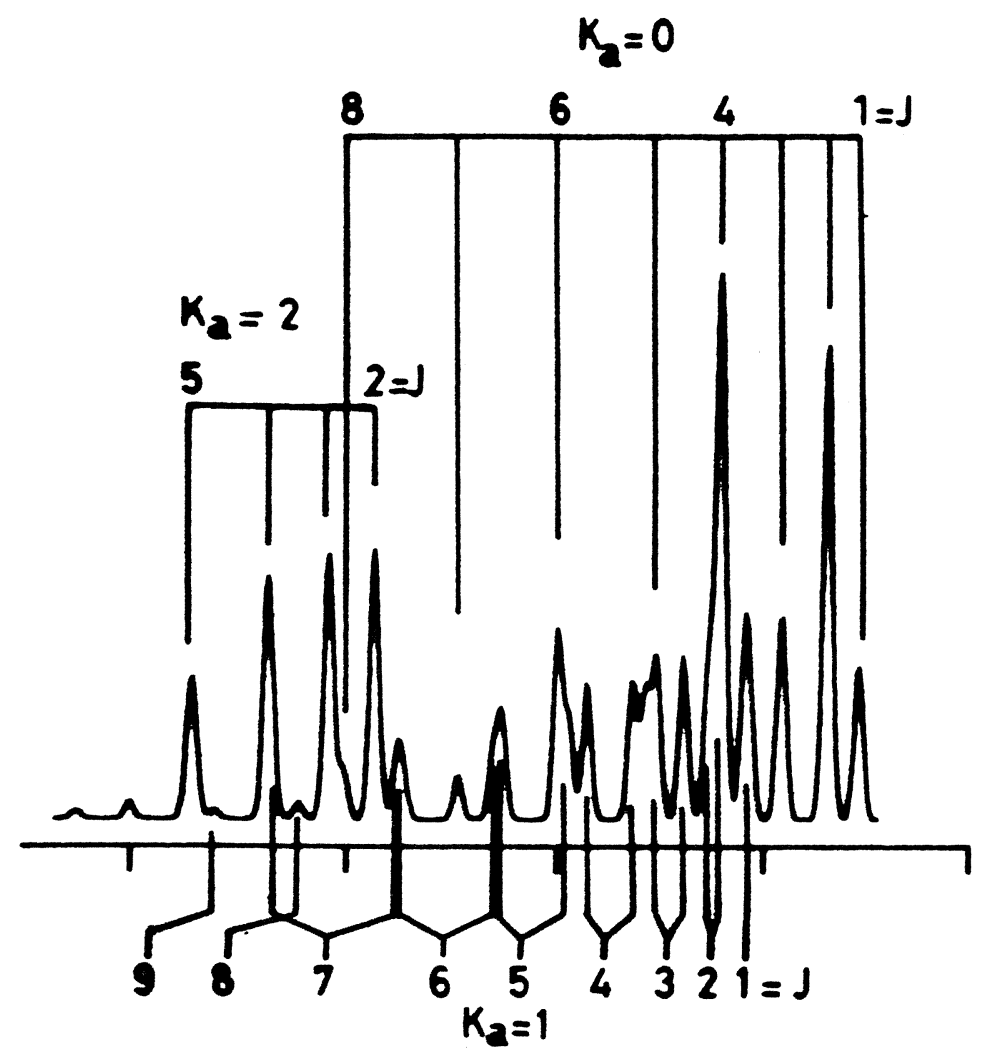

Exp.

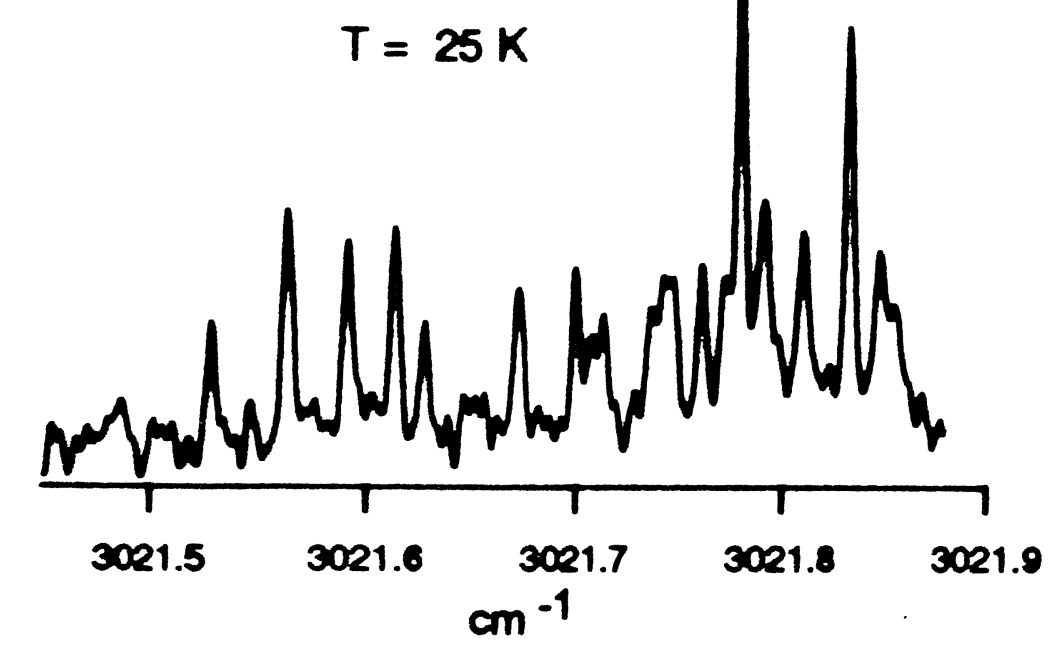

Calc.

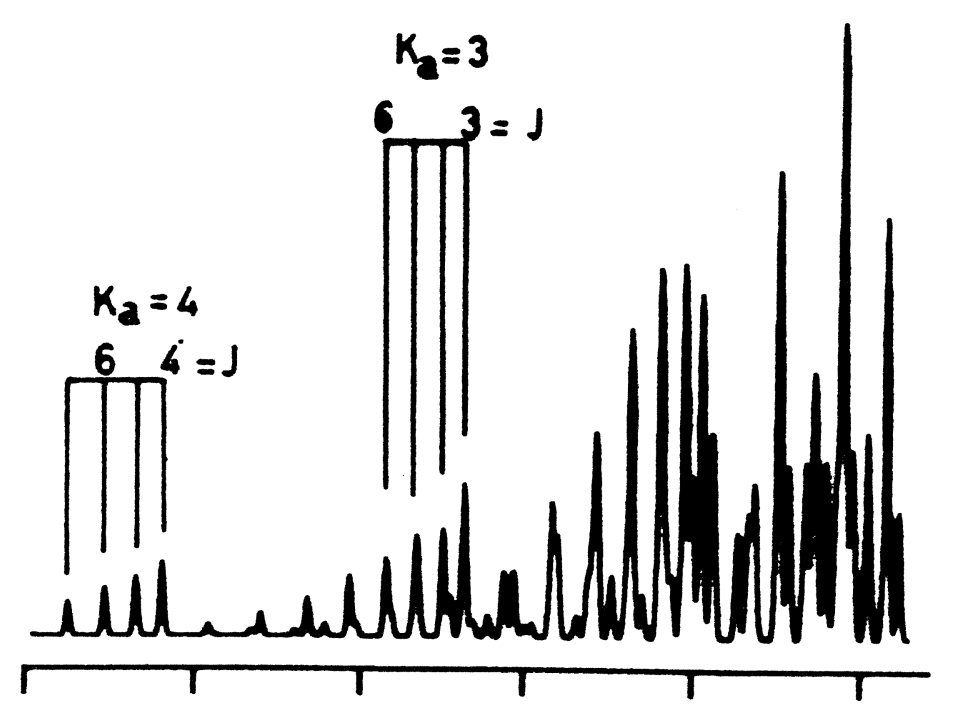

Exp.

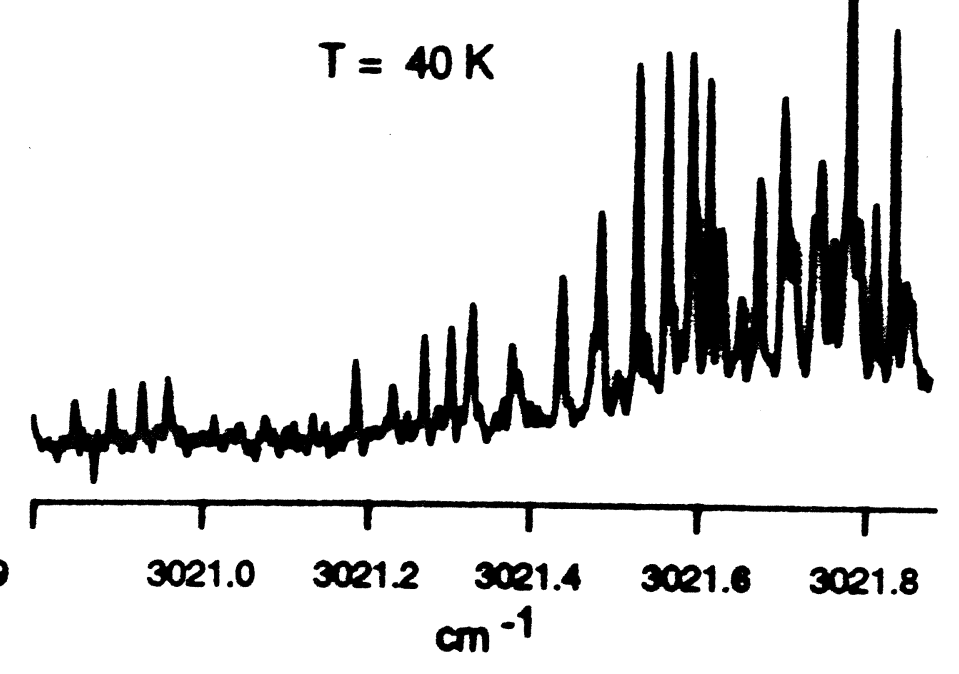




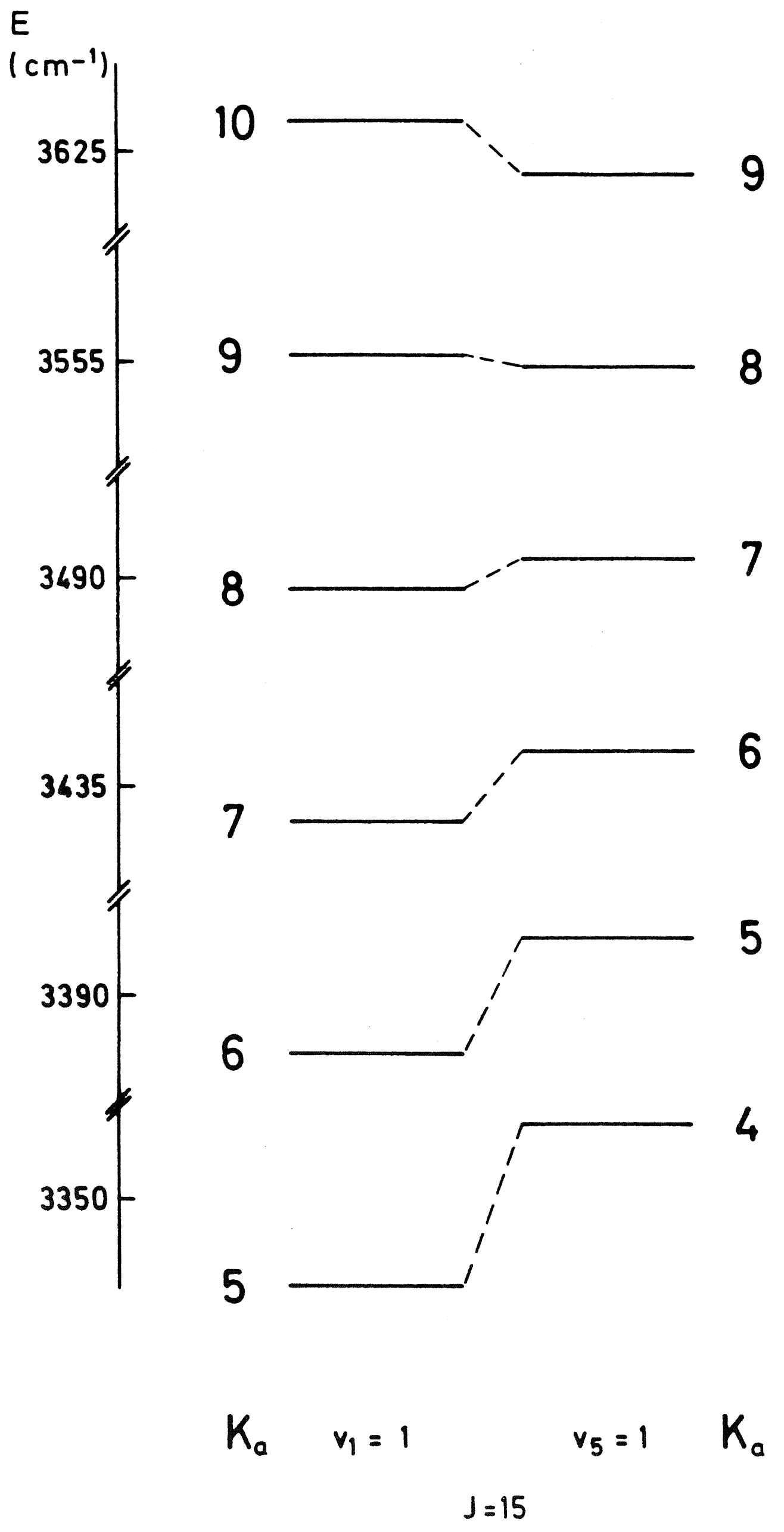

